\title{
Effects of Joint Cryotherapy on Muscle Function
}

\author{
Kyung-Min Kim \\ Department of Kinesiology and Sport Sciences, University of Miami, Coral Gables, FL, USA
}

PURPOSE: To summarize evidence regarding efficacy of focal joint cooling on muscle function.

METHODS: Literature review was performed to determine effectiveness of focal joint cooling on muscle function. Therapeutic cooling, professionally termed cryotherapy, has a long history in sports medicine because it has been widely used for a variety of therapeutic purposes. However, it has been contraindicated in rehabilitation for patients with muscle dysfunction because it is believed that cryotherapy has detrimental effects on muscle function. It is clinically important to recognize that the negative outcomes may result from the common mode of cryotherapy involving the direct cooling of muscle fibers. In contrast, there is promising evidence that when cryotherapy targets joints where muscle fibers are not located, the negative effects on muscle function can be eliminated or there can even be positive effects on muscle function.

RESULTS: Focal joint cooling appears to be effective in increasing motor neuron activation in patients with joint pathology in the lower extremity, leading to greater muscle strength. In addition, joint cryotherapy may be capable of negating deficiencies in functional performance while it was not found to be neither beneficial nor harmful to reflexive action and postural control.

CONCLUSIONS: Joint cryotherapy can be a safe and effective intervention for improving muscle function, and it should be indicated for patients with persistent muscle dysfunction.

Key words: Cryotherapy, Focal joint cooling, Muscle function

\section{INTRODUCTION}

Cryotherapy, therapeutic cooling, is one of the modalities widely used in sports medicine for a variety of treatment purposes. Physiological and neurological responses of musculoskeletal tissues to cooling have been extensively examined in the literature. It is thought that cryotherapy decreases temperature in skin, muscle, and/or intra-articular structures, and results in relief in clinical problems such as acute inflammatory processes, pain, swelling, muscle spasms, and symptoms of delayed-onset muscle soreness [1,2]. On the other hand, cryotherapy is believed to have detrimental effects on motor function, including motor nerve conduction velocity and synaptic transmission, muscle spindle sensitivity and firing rates, muscle strength, postural control, and functional performance [1-3]. In addition a recent review suggested that clinicians should be cautious in returning an athlete to dynamic activities immediately af- ter a cryotherapy treatment due to its detrimental effects on joint position sense [4].

There is ample anecdotal evidence supporting clinical uses of cryotherapy in sports medicine because of analgesic effects and reduction in secondary hypoxic cell death during immediate care of acute injury $[1,2]$. Multiple systematic reviews, however, found that the existing scientific evidence is insufficient to conclude whether this modality is actually effective in improving clinical outcomes such as reduced pain and swelling, improved function, and quicker return to participation in normal activity following acute soft tissue injury [3,5-9]. For example, a recent systematic review of 10 randomized controlled trials with a meta-analysis investigated the effectiveness of cryotherapy following anterior cruciate ligament reconstruction (ACL-R) on various outcomes, including pain, edema, and knee function [9]. For pain, the review authors performed a meta-analysis of two studies and determined that the use of a

Corresponding author: Kyung-Min Kim Tel +1-305-284-6959 Fax +1-305-284-4183 E-mail kmk154Amiami.edu

Received 22 Oct 2016 Revised 28 Nov 2016 Accepted 2 Jan 2017

(a) This is an Open Access article distributed under the terms of the Creative Commons Attribution Non-Commercial License (http://creativecommons.org/licenses/by-nc/4.0/) which permits unrestricted non-commercial use, distribution, and reproduction in any medium, provided the original work is properly cited. 
cold compression device (CCD) is superior to no cryotherapy (large effects found in visual analog scale pain score 48 hours after ACL-R). However, this favorable outcome is challenged by another 3 studies in that review reporting no differences when comparing CCD with cold water against $\mathrm{CCD}$ with room temperature water. For edema, one of 3 studies only reported a small improvement in patients using CCD compared to those receiving ice packs. Similarly, for knee function, one of 2 studies found a small improvement favoring CCD over ice packs. These conflicting results make it difficult to draw definitive conclusions on the effectiveness of cryotherapy although CCD appears to be effective in managing immediate post-surgery pain [9].

It is traditionally thought that cryotherapy is harmful to muscle function. However, it should be noted that negative effects following cooling therapy were mostly found during or after direct muscle cooling that stiffens muscle fibers, which results in decline in muscle function [1-3]. Interestingly, there is emerging scientific evidence that focal joint cooling may have the potential to improve neuromuscular function in that cooling a joint may not involve muscle fibers and appears to stimulate the central nervous system thus providing more neural drive to the neuromuscular junction [10-18]. Therefore, it is imperative to review the literature on joint cooling and muscle function and consider clinical use of focal joint cooling in rehabilitation for patients with muscle dysfunction. This paper will discuss the specific effects of joint cryotherapy on muscle function, including muscle activation and strength, reflexive reaction, postural control, and functional performance.

\section{Muscle Activation and Strength}

Numerous studies have assessed muscle activation and strength following joint cryotherapy in various joint conditions, including healthy, artificially effused, and pathological joints [10-23]. A majority of studies reported immediate facilitation in muscles that act on the cooled joint. This was primarily demonstrated through increased force outputs. For example, Hopkins et al. [11] assessed the effects of ankle joint cryotherapy on the soleus function over a 60-minute period in healthy ankle joints [11]. Specifically, the study assessed motor neuron activation, as measured with Hoffmann reflex and maximal voluntary isometric contraction (MVIC) during peak plantar flexion torque before and after 30 minutes of focal ankle joint cooling, and found that ankle cryotherapy significantly facilitated motor neuron activation and substantially increased MVIC. These immediate effects were sustained over a 60 -minute rewarming period. More importantly, changes in the motor neuron activation and MVIC were significantly correlated $(\mathrm{r}=.38)$, suggesting that cooling the skin over the ankle joint might cause a systemic or centrally mediated change in excitability or threshold level at the motoneuron pool. This change, in turn, could lead to more neural drive to the neuromuscular junction, which can explain increased MVIC following joint cooling. Subsequent studies reported similar findings of facilitated motor neuron activation in ankle muscles [12,18-22].

In addition to ankle cryotherapy, focal knee joint cooling was examined to determine quadriceps function in healthy knee joints [14]. Quadriceps function was assessed with peak knee extension torque and voluntary activation during maximal isometric knee extension. Central activation ratio was the measure of voluntary activation, calculated by dividing force measurements of maximal voluntary contraction by that of the force produced by superimposed burst plus the maximal voluntary contraction. Significantly increased voluntary activation and peak knee extension torque were found following 20-minute of knee joint cryotherapy, and changes in maximal voluntary contractions explained a significant amount of the variance in the activation. These results show that the increase in voluntary contractions may be due to an increase in the volitional activation, resulting from the facilitated motor neurons previously found [11].

Apart from the promising effects of joint cryotherapy on healthy joint function, cooling has been applied to artificially effused and pathological joints in efforts to determine its therapeutic effects Hopkins et al. [10] assessed effects of knee joint cryotherapy on motor neuron activation in healthy subjects with simulated knee effusion that has been found to cause decrease in quadriceps neural activation [10]. The knee joint cryotherapy resulted in significant dis-inhibition as well as facilitation of the quadriceps activation. It is noted that there are different interpretations between disinhibition and facilitation. Dis-inhibition can be viewed as a recovery of decreased motor neuron activation to a pre-effusion level while facilitation is characterized by additional increase in motor neuron activation compared to the pre-effusion level. The facilitation of motor neurons has been manifested following joint cryotherapy in healthy joints. In the effused knee, these similar facilitating effects continued to increase for up to 60 minutes even after the ice was removed. Another study investigated the effects of knee cryotherapy on quadriceps peak torque following a simulated knee effusion, and found knee extension torque significantly decreased following knee effusion, but this reduction was recovered following knee joint cryotherapy [15]. Authors interpret these findings as clear evidence that quadriceps inhibition created by 
knee effusion is due to altered sensory information from the mechanoreceptors within the knee joint $[10,15]$. Cooling the effused knee seems to freeze the altered sensory information that is responsible for inhibition, leading to decrease in inhibition (dis-inhibition), as expressed with increase in motor neuron activation and peak knee extension torque $[10,15]$.

The favorable outcomes following joint cryotherapy in effused joints triggered initiation of clinical studies assessing effects of joint cryotherapy for patients with joint pathology in the lower extremity. Pietrosimone et al. [24] investigated the effects of focal knee joint cooling on quadriceps activation in patients with tibiofemoral osteoarthritis who have been found to have voluntary quadriceps activation deficits reflecting arthrogenic muscle inhibition [24]. The focal knee joint cooling consisted of two 1.5 L bags of crushed ice secured to the anterior and the posterior aspects of the knee joint with an elastic bandage for 20 minutes. The knee cryotherapy was found to immediately dis-inhibit or facilitate quadriceps voluntary activation, and its effects were sustained after the ice bags were removed. In addition, peak knee extension torque tended to increase following the ice treatment, but did not reach a statistically significant difference. However, it should be noted that the variance in the changes in the quadriceps strength explained a significant amount of the variance $\left(\mathrm{r}^{2}=.81\right)$ in the change in central activation ratio, suggesting that the slight increase in muscle strength may be due to significant increase in neural drive from the central nervous system.

In addition to knee cryotherapy, ankle cryotherapy has been examined in patients with chronic ankle instability (CAI). In two investigations, Doeringer et al. $[19,20]$ assessed the effects of focal ankle joint cooling on motor neuron activation of lower leg muscles, including the soleus, peroneus longus, and tibialis anterior in individuals with and without CAI. The ankle cryotherapy consisted of one $1.5 \mathrm{~L}$ bag of crushed ice secured to the anterior aspect of the ankle joint with an elastic bandage for 30 minutes. The soleus motor neuron activation was facilitated following the treatment in both healthy and CAI groups, but there was no group difference, indicating no additional facilitating effects [19]. The same group also reported a lack of change in other outcomes such as motor neuron activations of the peroneus longus and tibialis anterior, and concentric inversion and eversion torques [20]. However, these conclusions may be inaccurate due to the unclear statistical analysis and the moderate effect sizes found in motor neuron activation of the peroneus longus and tibialis anterior between pre- and post-cooling. For these limitations, Kim et al. [18] recently conducted another study to examine whether focal ankle joint cooling affects motor neuron activations of lower leg muscles in individuals with and without CAI They found that ankle cryotherapy for 20 minutes significantly facilitated motor neuron activation of soleus and peroneus longus in individuals both with and without CAI, without group differences [18]. These results support the notion that motor neurons in muscles surrounding healthy and pathological ankle joints would respond similarly to joint cryotherapy. Furthermore, the study found that the facilitating effects ankle cryotherapy has when subjects were at rest disappeared when they moved from lying to standing positions (bipedal and unipedal stances) [18]. This implies that patients with decreased motor neuron activation may only benefit from joint cryotherapy that is applied to patients at rest. Collectively, joint cryotherapy can be a promising tool to maximize muscle activation and strength for patients with joint pathology. It is suggested to use joint cryotherapy prior to exercise to have the opportunity to utilize increased motor neuron activation that provides more neural drive to the neuromuscular junction, resulting in greater muscle strength $[18,23,25]$.

\section{Reflexive Reaction}

A reflexive reaction of joint stabilizing muscle is a critical component of joint stability [26,27]. These reactions have been considered as the first line of protection against joint injury. In the ankle literature, reaction time of the peroneals to inversion perturbation has been extensively examined in individuals with a history of ankle sprains because delayed onset of peroneal activation in response to inversion perturbation may be one of reasons of subjective ankle instability and recurrent injury in people with a history of ankle sprains [26-28].

It is thought that reflexive reactions are impaired following cryotherapy because of convincing evidence that the reflex latencies of human Achilles tendons and rabbit patellar tendons are significantly decreased following ice treatment $[29,30]$. However, these findings were observed following direct muscle cooling. Cooling a joint may have differential effects on a reflexive behavior as it contributes to motoneuron recruitment and force production [31-33]. Studies have been conducted to assess peroneal reactions to sudden inversion following ankle joint cryotherapy. All of the studies reported consistent findings that the peroneal reaction was not impaired following ankle joint cooling [31-33]. Furthermore, it has been demonstrated that focal knee joint cooling did not affect the hamstring stretch reflex induced by anterior tibia translation [34]. These findings suggest that joint cryotherapy may be a safe modality for those 
who return to play following ice treatment for a variety of purposes, predominantly pain relief. However, caution should be used when generalizing findings from healthy subjects into those with injuries.

\section{Postural Control}

There are few studies that have assessed the effects of cryotherapy on postural control, as measured with instrumented balance tests. The limited research may be due to previous reports of decreased muscle spindle sensitivity following ice treatment $[29,35]$. Balance performance has been assessed following immersion cryotherapy of the ankle/foot complex in healthy subjects and patients with lateral ankle sprains [36,37]. Both subject groups demonstrated impaired single-leg balance after cooling. It should be noted that the ankle was cooled along with lower leg muscles in both studies, which likely decreased muscle spindle sensitivity in cooled muscles, thus resulting in postural impairment.

In contrast to cooling the ankle/foot complex, application of cold only to the ankle joint has been also examined [38,39]. Roberts first investigated the effects of ankle cryotherapy on single-leg balance in 30 healthy subjects [39]. The outcome measure was the amount of sway in a mediolateral direction during unipedal balance. Interestingly, a one-tailed t-test was used to compare pre-ice with post-ice sway data for each subject, meaning 30 t-tests were used for the 30 subjects. Single-leg balance performance was significantly diminished after ice treatment in 17 subjects while it substantially improved in 11, and balance performance was unchanged in the remaining 2 subjects. These findings may be not accurate due to poor quality of study design including a lack of a control group, use of unusual statistical approaches, and a lack of multiple trials of balance tests. Kim et al. [38] recently conducted a follow-up study to confirm the previous findings with a stronger study design, and found that ankle cryotherapy did not change single-leg balance performance. The authors speculated the lack of change in single-leg balance performance following focal ankle joint cooling may be attributed to redundancy of proprioceptive information from other receptors besides the ankle, a potential involvement of intrinsic foot muscles, and lack of icing influences on muscle spindles that are typically located in the muscle belly that is outside the cooled area of the joint $[40,41]$.

The literature is lacking clear evidence regarding the effects of joint cryotherapy on postural control, which is a critical aspect of injury management. Application of an ice bag to an injured joint is the most common mode of cryotherapy in the acute stage of injury care in sports medicine, and focal joint cooling becomes more popular in rehabilita- tion for those with knee or ankle pathologies due to emerging evidence for the clinical efficacy of joint cryotherapy on muscle activation and force production. It has been consistently documented that postural control is impaired following a joint injury, which is associated with a higher risk of lower extremity injury. Therefore, it is of paramount importance to discover whether joint cryotherapy affects postural control in people who are healthy, acutely injured, and those with chronic joint problems.

\section{Functional Performance}

There are conflicting results about the effects of joint cryotherapy on functional performance [42-46]. Hart et al. [42] evaluated changes in ground reaction forces, knee-joint flexion, and muscle activity of multiple muscles in the lower extremity during single-leg landings after 20-minute knee joint cryotherapy, and found the treatment did not change any of these measures. It was also found that peak torque, peak power, and root mean square power from the ankle, knee, and hip joints during a semi-recumbent stepping motion on closed-chain dynamometer remained unchanged following either ankle or knee joint cooling [43] Furthermore, ankle cryotherapy has not been found to change ankle kinematics during a sidestep cutting maneuver [44]. These studies suggest that joint cryotherapy may be a safe treatment that would not inhibit the normal motor function that dynamically supports a joint. However, it was reported that leg muscle activity was diminished during a single-leg drop jump after cooling the knee joint, suggesting joint cryotherapy is contraindicated for those who return to play or competition [46].

There have been interesting findings regarding the therapeutic potential of joint cryotherapy to improve functional performance in individuals with artificial quadriceps inhibition. Hopkins investigated the effects of knee cryotherapy on functional activities in the knee joint with quadriceps inhibition that was artificially induced with joint effusion [45] There were three subject groups in that study: a group without joint effusion and with the ice treatment (control), another with joint effusion but without the treatment (effusion), and the other with both the joint effusion and treatment (cryotherapy). There was a significant decrease in peak torque and peak power in the effusion group whereas no change was observed over time in the control and cryotherapy groups. It was also found that the peak activity in the vastus lateralis muscle of the effusion group was significantly decreased when compared to peak activity of other groups, while the cryotherapy group presented a greater knee anterior joint reaction force than reaction force observed in other 
groups. These findings showed that knee cryotherapy negated movement deficiencies, which may be due to the expected facilitating effects that cooling has on vastus lateralis activation.

\section{CONCLUSION}

Joint cryotherapy may have the potential to be added to muscle strength rehabilitation programs in patients with joint pathology in the lower extremity. It is thought that cryotherapy is an effective modality not only to reduce pain, swelling, and secondary hypoxic injury during immediate care, but also to induce analgesic effects during the early stage of rehabilitation to facilitate the initiation of active therapeutic exercise. In contrast, this therapy has been contraindicated during rehabilitation for muscle function due to detrimental effects such as decreased motor nerve conduction velocity, muscle spindle sensitivity, and muscle strength. However, it is important to note that these negative effects were found following direct muscle cooling, while there is growing evidence indicating improved muscle function following focal joint cooling. Specifically, joint cryotherapy has been found to increase motor neuron activation in patients with joint pathology in the lower extremity, leading to greater muscle strength. Joint cryotherapy has also negated deficiencies in functional performance. Furthermore, this therapy has not been found to have deleterious effects on other muscle functions such as reflexive action and postural control. These results suggest that joint cryotherapy can be a safe and effective intervention for improving muscle function, and it should be indicated for patients with persistent muscle dysfunction.

\section{REFERENCES}

1. Swenson C, Sward L, Karlsson J. Cryotherapy in sports medicine. Scandinavian Journal of Medicine and Science in Sports 1996;6(4): 193-200.

2. Knight K. Cryotherapy in Sport Injury Management. Champaign: Human Kinetics; 1995.

3. Collins NC. Is ice right? Does cryotherapy improve outcome for acute soft tissue injury?. Emergency Medicine Journal 2008;25(2):65-68.

4. Costello JT, Donnelly AE. Cryotherapy and joint position sense in healthy participants: a systematic review. Journal of Athletic Training 2010;45(3):306-316.

5. Bleakley C, McDonough S, MacAuley D. The use of ice in the treat- ment of acute soft-tissue injury: a systematic review of randomized controlled trials. The American Journal of Sports Medicine 2004;32(1) 251-261

6. Hubbard TJ, Denegar CR. Does Cryotherapy Improve Outcomes With Soft Tissue Injury?. Journal of Athletic Training 2004;39(3):278279.

7. Hubbard TJ, Aronson SL, Denegar CR. Does Cryotherapy Hasten Return to Participation? A Systematic Review. Journal of Athletic Training 2004;39(1):88-94.

8. French SD, Cameron M, Walker BF, Reggars JW, Esterman AJ. A Cochrane review of superficial heat or cold for low back pain. Spine 2006;31(9):998-1006.

9. Martimbianco AL, Gomes da Silva BN, de Carvalho AP, Silva V, Torloni MR, et al. Effectiveness and safety of cryotherapy after arthroscopic anterior cruciate ligament reconstruction. A systematic review of the literature. Physical Therapy in Sport 2014;15(4):261-268.

10. Hopkins JT, Ingersoll CD, Edwards J, Klootwyk TE. Cryotherapy and transcutaneous electric neuromuscular stimulation decrease arthrogenic muscle inhibition of the vastus medialis after knee joint effusion. Journal of Athletic Training 2002;37(1):25.

11. Hopkins JT, Stencil R. Ankle cryotherapy facilitates soleus function. Journal of Orthopaedic and Sports Physical Therapy 2002;32(12):622627.

12. Palmieri-Smith RM, Leonard-Frye JL, Garrison CJ, Weltman A, Ingersoll CD. Peripheral joint cooling increases spinal reflex excitability and serum norepinephrine. International Journal of Neuroscience 2007; 117(2):229-242.

13. Pietrosimone BG, Hart JM, Saliba SA, Hertel J, Ingersoll CD. Immediate effects of transcutaneous electrical nerve stimulation and focal knee joint cooling on quadriceps activation. Medicine and Science in Sports and Exercise 2009;41(6):1175-1181.

14. Pietrosimone BG, Ingersoll CD. Focal knee joint cooling increases the quadriceps central activation ratio. Journal of Sports Sciences 2009; 27(8):873-879.

15. Rice DA, McNair PJ, Dalbeth N. Effects of cryotherapy on arthrogenic muscle inhibition using an experimental model of knee swelling. Arthritis Care \& Research 2009;61(1):78-83.

16. Hart JM, Kuenze CM, Diduch DR, Ingersoll CD. Quadriceps muscle function after rehabilitation with cryotherapy in patients with anterior cruciate ligament reconstruction. Journal of Athletic Training 2014; 49(6):733-739 
17. Ewell M, Griffin C, Hull J. The use of focal knee joint cryotherapy to improve functional outcomes after total knee arthroplasiy: Review. PM\&R 2014;6(8):729-738.

18. Kim KM, Ingersoll CD, Hertel J. Facilitation of hoffmann reflexes of ankle muscles in prone but not standing positions by focal ankle-joint cooling. Journal of Sport Rehabilitation 2015;24(2):130-139.

19. Doeringer JR, Hoch MC, Krause BA. The effect of focal ankle cooling on spinal reflex activity in individuals with chronic ankle instability. Athletic Training \& Sports Health Care. 2009;1(2):59-64.

20. Doeringer JR, Hoch MC, Krause BA. Ice application effects on peroneus longus and tibialis anterior motoneuron excitability in subjects with functional ankle instability. The International Journal of Neuroscience 2010;120(1):17-22.

21. Krause BA, Hopkins JT, Ingersoll CD, Cordova ML, Edwards JE. The relationship of ankle temperature during cooling and rewarming to the human soleus H reflex. Journal of Sport Rehabilitation 2000;9(3): 253-262.

22. Kim KM, Ingersoll CD, Hertel J. Facilitation of hoffmann reflexes of ankle muscles in prone but not standing positions by focal ankle-joint colling. Journal of Sport Rehabilitation 2015;24(2):130-139.

23. Harkey MS, Gribble PA, Pietrosimone BG. Disinhibitory interventions and voluntary quadriceps activation: a systematic review. Journal of Athletic Training 2014;49(3):411-421.

24. Pietrosimone BG, Hertel J, Ingersoll CD, Hart JM, Saliba SA. Voluntary quadriceps activation deficits in patients with tibiofemoral osteoarthritis: a meta-analysis. PM\&R 2011;3(2):153-162.

25. Palmieri-Smith RM, Thomas AC, Wojtys EM. Maximizing quadriceps strength after ACL reconstruction. Clinics in Sports Medicine 2008; 27(3):405-424.

26. Khin Myo H, Ishii T, Sakane M, Hayashi K. Effect of anesthesia of the sinus tarsi on peroneal reaction time in patients with functional instability of the ankle. Foot \& Ankle International 1999;20(9):554-559.

27. Konradsen L, Ravn JB. Ankle instability caused by prolonged peroneal reaction time. Acta Orthopaedica Scandinavica 1990;61(5):388-390.

28. Hopkins JT, Brown TN, Christensen L, Palmieri-Smith RM. Deficits in peroneal latency and electromechanical delay in patients with functional ankle instability. Journal of Orthopaedic Research 2009;27(12): 1541-1546.

29. Bell KR, Lehmann JF. Effect of cooling on H- and T-reflexes in normal subjects. Archives of Physical Medicine and Rehabilitation 1987; 68(8):490-493.
30. Lee SU, Bang MS, Han TR. Effect of cold air therapy in relieving spasticity: applied to spinalized rabbits. Spinal Cord 2002;40(4):167-173.

31. Cordova ML, Bernard LW, Au KK, Demchak TJ, Stone MB, et al. Cryotherapy and ankle bracing effects on peroneus longus response during sudden inversion. Journal of Electromyography and Kinesiology 2010;20(2):348-353.

32. Berg CL, Hart JM, Palmieri-Smith R, Cross KM, Ingersoll CD. Cryotherapy does not affect peroneal reaction following sudden inversion. Journal of Sport Rehabilitation 2007;16(4):285-294.

33. Hopkins JT, Hunter I, McLoda T. Effects of ankle joint cooling on peroneal short latency response. Journal of Sports Science and Medicine. 2006;5(2):333-339

34. Melnyk M, Faist M, Claes L, Friemert B. Therapeutic cooling: no effect on hamstring reflexes and knee stability. Medicine and Science in Sports and Exercise 2006;38(7):1329-1334

35. Eldred E, Lindsley DF, Buchwald JS. The effect of cooling on mammalian muscle spindles. Experimental Neurology 1960;2(2):144-157.

36. Kernozek TW, Greany JF, Anderson DR, Van Heel D, Youngdahl RL, et al. The effect of immersion cryotherapy on medial-lateral postural sway variability in individuals with a lateral ankle sprain. Physiotherapy Research International 2008;13(2):107-118.

37. Weimar W, Campbell B. The influence of ankle cryotherapy on unilateral static balance. Medicine and Science in Sports and Exercise 2004;36(5):S187.

38. Kim KM, Hart JM, Saliba SA, Hertel J. Effects of focal ankle joint cooling on unipedal static balance in individuals with and without chronic ankle instability. Gait and Posture 2015;41(1):282-287.

39. Roberts ML. The effect of Ice pack application at the ankle joint on one-legged balance ability. New Zealand Journal of Physiotherapy 1994;22:17-22.

40. Konradsen L, Ravn JB, Sorensen AI. Proprioception at the ankle: the effect of anaesthetic blockade of ligament receptors. Journal of Bone and Joint 1993;75(3):433-436.

41. Riemann BL, Myers JB, Stone DA, Lephart SM. Effect of lateral ankle ligament anesthesia on single-leg stance stability. Medicine and Science in Sports and Exercise 2004;36(3):388-396.

42. Hart JM, Leonard JL, Ingersoll CD. Single-leg landing strategy after knee-joint cryotherapy. Journal of Sport Rehabilitation 2005;14(4):313320.

43. Hopkins J, Adolph JT. Effects of joint cryotherapy on lower chain function. Clnical Kinesiology 2003;57(3):42-48. 
44. Atnip BL, McCrory JL. The effect of cryotherapy on three dimensional ankle kinematics during a sidestep cutting maneuver. Journal of Sports Science and Medicine 2004;3(2):83-90.

45. Hopkins JT. Knee joint effusion and cryotherapy alter lower chain kinetics and muscle activity. Journal of Athletic Training 2006;41(2):177-
184.

46. Schmid S, Moffat M, Gutierrez GM. Effect of knee joint cooling on the electromyographic activity of lower extremity muscles during a plyometric exercise. Journal of Electromyography and Kinesiology 2010;20(6):1075-1081. 